\title{
Lung Cancer Tumor Detection using Image Processing and Bounding Box
}

\author{
Vishal Gundavarapu $^{\# 1}$, S. Srivatsan ${ }^{\# 2}$, Shrnjay Krishnan. $V^{\# 3}$ \\ \#1,\#2,\#3 School of electrical engineering, Vellore Institute of Technology,Chennai- \\ 600127, Tamil Nadu, India.
}

\section{ABSTRACT}

Image processing has been successfully used for detection and identification of certain elements by enhancing the features of a tumour. This paper focuses on using image processing techniques to isolate the location of a tumour and use a bounding box algorithm to effectively detect cancerous tumours, by using image binarization and image segmentation algorithms to detect cancerous tumours by processing the CAT scans and XRays.

Key words:Binarization, Feature Extraction Image Acquisition, Image Pre-processing, Segmentation, Thresholding.

\section{INTRODUCTION}

Lung cancer is by far the top cause of cancer deaths among people throughout the world. It hasthehighest mortality rate among all other type of cancers. The chances of survival greatly increase if the cancer is detected in its early stages. Therefore, the need for detecting the lung nodule at early stage in CT medical images arises. To predict the lung cancer, various features are extracted from the image. Our application performs steps such as acquiring the image, pre-processing, converting the image to black and white using thresholding, segmenting the lung as required, extracting features of tumours and detection using a bounding box algorithm.

There are four stages of lung cancer. Staging is based on tumour size and tumour \& lymph node location. The survival rate for all the stages of lung cancer combined has not improved in spite of treatment procedures like surgery, radiation therapy, chemo therapy etc. Therefore, the paper aims to increase the detection of lung cancer by using automated means to see all probabilities of cancer in the lung.

\section{STEPS INVOLVED}

\section{Acquiring Image}

Generally, a Computed Tomography scan is used in the case of checking for lung tumours as they can show the size, shape and location of the tumour on the lung. We have collected CT scans of normal and cancerous lungs from the internet.

\section{Image Pre-processing}

After obtaining the image of the lung as a CT scan, the scans are passed through Image Preprocessing stage. The steps involved in this stage are:

- Greyscale conversion 
- Normalization

- Noise reduction

- Converting to binary image

- Removing unwanted portions

\section{Conversion to binary image}

The CT scan is first converted to a grey scale image. The noise in the grey scale image is removed using the command medfilt2. The grey scale image is then converted to a binary image. This step is called binarization. A binary image, as the name suggests, has two colours, black and white, where black has a value of 0 and white has a value of 1 .

\section{Segmenting the image}

This is the most important step in this application. After this step, the tumour can be visualized clearly. Segmenting involves processes such as conversion to edge only image which helps to enhance the boundaries of the lung and the cancer cells. The edges are then dilated to further enhance the tumour's features. The dilated image is then filled to remove unnecessary spots. Then, we locate the boundaries of the two lungs from the CT scan. The image is then segmented into left lung and right lung in order to undergo the bounding box algorithm.

\section{Thresholding}

A threshold value is required to turn a greyscale image into a black and white (binary) image. Depending on the 'greyness' and the threshold value, a pixel will be converted to either black or white. The primary focus was to find a threshold value which can be applicable in any case. Therefore, we arrived at a value after experimentation on trial and error basis.

\section{Feature Extraction}

After all the image preprocessing, we are left with the lung with a well demarcated tumour (if it is present). This is the final stage of extraction. Since a tumour is a lump of cells, the image is processed in a way such that any location with high cell density gets shown in white.

\section{Bounding Box Detection}

After the feature extraction process, the resulting image is then made to go through a bounding box algorithm, which predicts the location of high-density cells based on the "whiteness" of a location. This is generally reliable. In case of multiple white spaces, the application prompts the user if they want to see more.

\section{WORKING}

\subsection{Image Processing}

Resizing the image

The image from the CT scan is resized using the imresize function in Matlab. A 140x200 pixel image is sufficient for further processing. Reducing the size helps in improving processing speed.

\section{Conversion to grayscale}

The RGB image obtained from the CT scan is converted to grayscale using the rgb2gray function. The image is converted to grayscale so that it can easily be converted to a binary image later. 


\section{Filtering noise}

To filter noise, a 2D median filter, medfilt2, is used. It reduces noise and preserves edges.

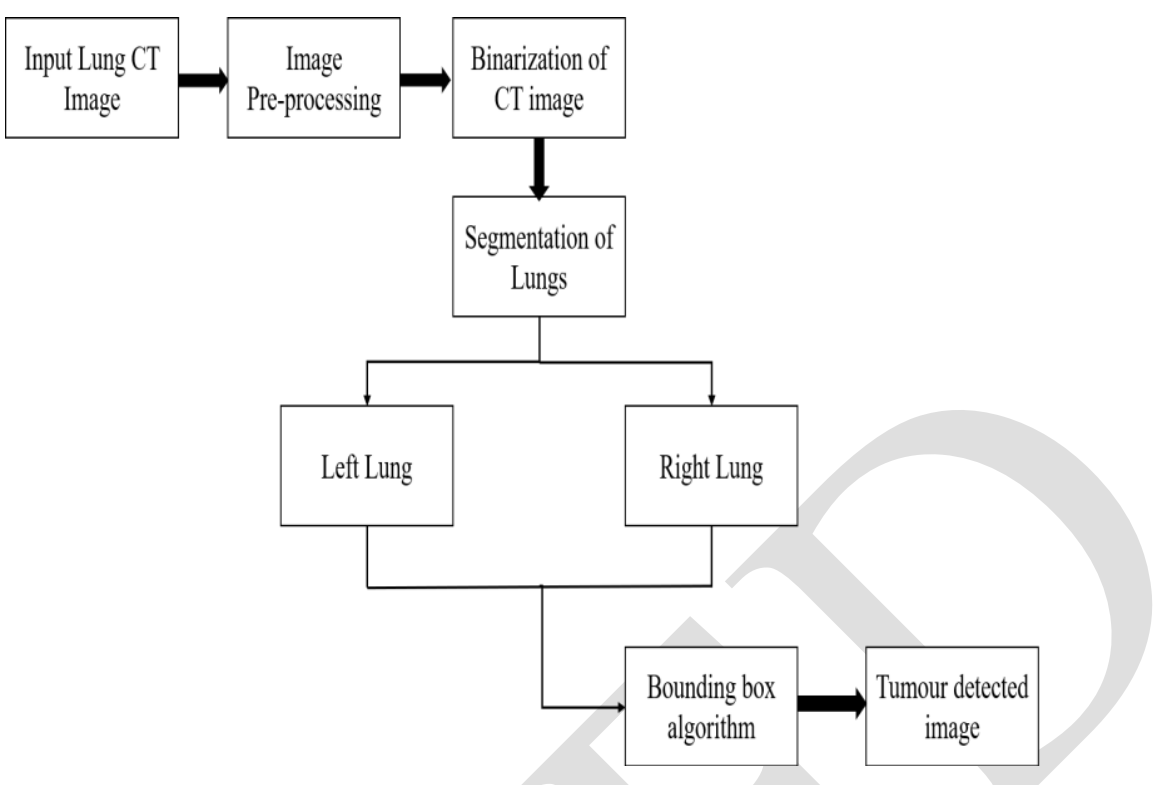

Figure 1: Flow chart of the processes

\section{Dilation}

To enhance the tumour and minimize the presence of other elementary particles, such as lung nodules, we use the imdilate function. In this case, a vertical line structuring element works best.

Fill

The dilated image is then filled to make segmentation of the lung easier.

\subsection{Segmentation}

After all the initial image processing, the image is divided into two parts, the left lung and right lung by roughly detecting the farthest edge of the left lung to the center and the closest edge of the right lung to the center and then dividing the image with respect to these points. The command bwareafilt is used to then further remove white spots that are not tumours.

\section{4. $\mathrm{CODE}$}

clc

clear all

img=imread('test2.jpg');

img=imresize(img,[140 200]);

img=rgb2gray(img);

img $=$ medfilt 2 (img);

img =im2 bw (img,0.55);

element 1 = strel('line', 2, 90);

element2 = strel('line', 2, 180);

img=edge(img);

img=imdilate(img, [element 1 element2]);

img=imfill(img,[90 120]); 


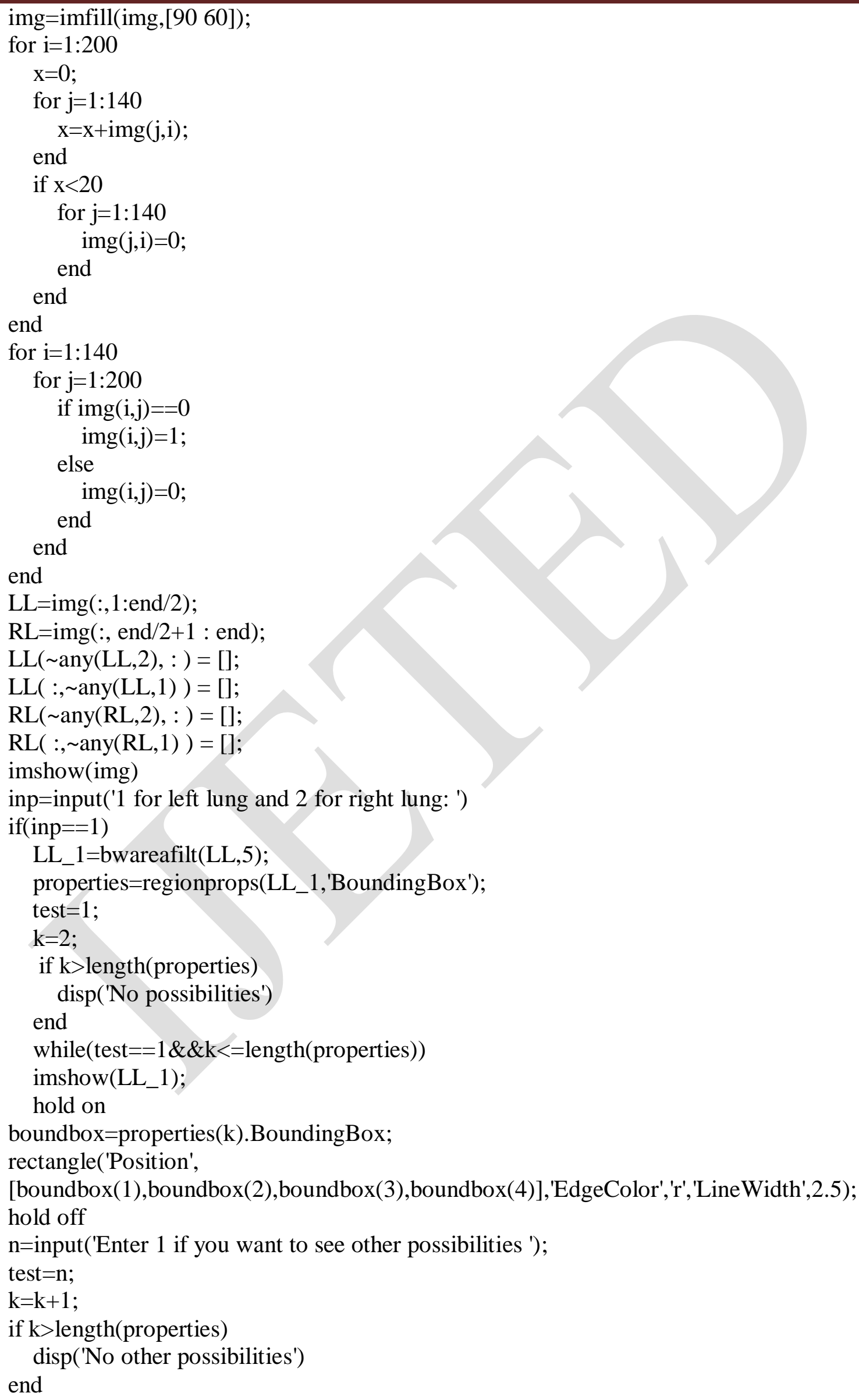




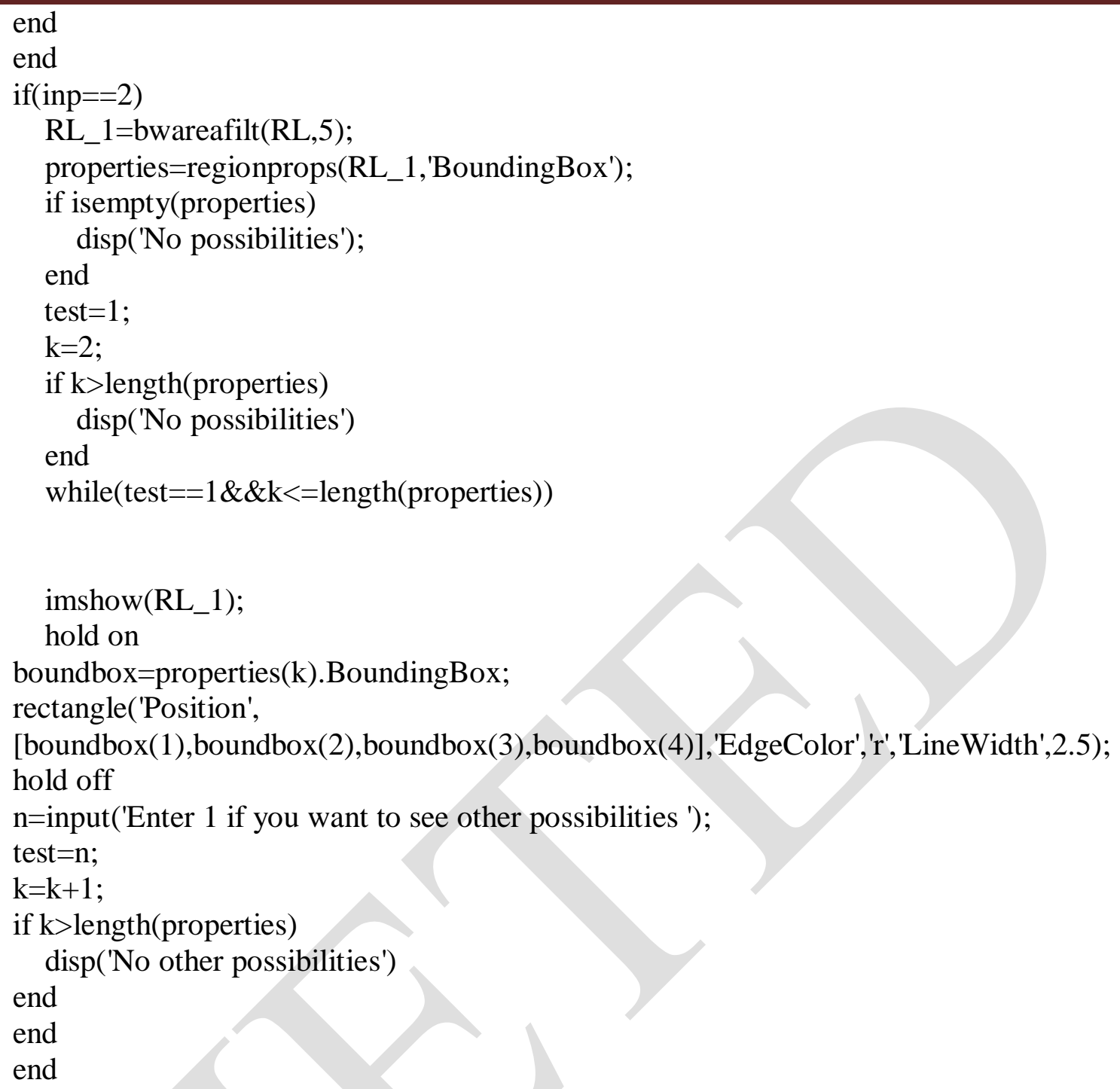

\section{RESULTS}

The following images are resulting outputs from the system. A bounding box is drawn around the suspected tumour location, which can be seen in the following pictures.

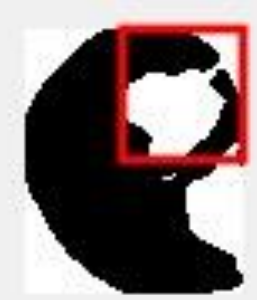

Figure 2: Lung sample 1

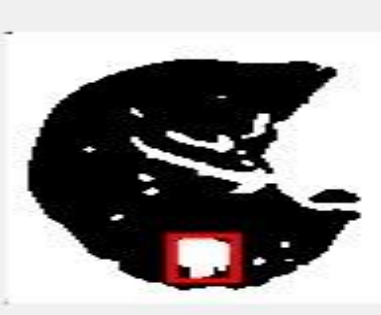

Figure 3: Lung sample 2

\section{CONCLUSION}

Due to increased effects of pollution, the cases of lung cancer have been rising. It is known to have the third highest mortality rate. Like with any other form of cancer, the chances of survival drastically increase if the tumour is detected sooner. Therefore, it seems sound to invest in machines that can efficiently detect cancer, as a result there is no room for human 
error. We have designed a system to detect lung tumour from CT Scans using image processing techniques in MATLAB, which is a high-performance tool for computing, and implemented a bounding box algorithm to improve the accuracy of the results.

\section{REFERENCES}

I. Padmanabhan Subbulakshmi, V.Sumathi*, S.Ganapathy, "Cloud-based POS System for Secured Smart Shopping CART Using RFID", Journal of Advanced Research in Dynamical and Control Systems Vol. 9. Sp- 14, 2017.

II. D. Lin and C. Van, "Lung nodules identification rules extraction with neural fuzzy network ", IEEE, Neural Information Processing, vol. 4, 2002.

III. Padmanabhan Subbulakshmi, "Arduino based Animal Intrusion Prevention System", International Journal of Emerging Trends in Engineering and Development Issue 9, Vol.1 2019.

IV. Md. Badrul Alam Miah and Mohammad Abu Yousuf, "Detection of Lung Cancer from CT Image Using Image Processing and Neural Network", 2nd International Conference on Electrical Engineering and Information \& Communication Technology (ICEEICT) 2015.

V. ARM Processor Based Vehicle Theft Detection System, Padmanabhan Subbulakshmi, MCharan Tej, M Manojsreenivas Reddy, International Journal of Emerging Trends in Engineering and Development Issue 9, Vol.1 2019.

VI. Anita Chaudhary and Sonit Sukhraj Singh, "Lung cancer detection on CT images by using image processing”, 2012 International Conference on Computing Sciences. 